\title{
REVIEW ARTICLE MAIT cells, guardians of skin and mucosa?
}

\author{
Isabelle Nel ${ }^{1,2}$, Léo Bertrand $\mathbb{D}^{1,2}$, Amine Toubal $\mathbb{D}^{1,2}$ and Agnès Lehuen (iD)
}

Mucosal Associated Invariant T (MAIT) cells are evolutionary conserved innate-like T cells able to recognize bacterial and fungal ligands derived from vitamin B biosynthesis. These cells are particularly present in liver and blood but also populate mucosal sites including skin, oral, intestinal, respiratory, and urogenital tracts that are in contact with the environment and microbiota of their host. Growing evidence suggests important involvement of MAIT cells in safeguarding the mucosa against external microbial threats. Simultaneously, mucosal MAIT cells have been implicated in immune and inflammatory pathologies affecting these organs. Here, we review the specificities of mucosal MAIT cells, their functions in the protection and maintenance of mucosal barriers, and their interactions with other mucosal cells.

Mucosal Immunology (2021) 14:803-814; https://doi.org/10.1038/s41385-021-00391-w

\section{INTRODUCTION}

The surface barriers of the organism separate the internal (self) from the external environment (non-self). These physical barriers include the skin and the mucosal barriers of the mouth, respiratory, gastrointestinal, and urogenital tracts. They are selectively permeable, allowing the passage of water, ions, nutrients, and gases while preventing entry of pathogens and toxins.

Protection against these threats is notably mediated by innate and adaptative immune cells that populate in important numbers mucosae and skin. These tissues are particularly enriched in innate cells as well as innate-like T cells, such as $\gamma \delta$ T cells, invariantNatural Killer T (iNKT) cells, and Mucosal Associated Invariant T (MAIT) cells that can, in the event of infection, produce various cytokines, mediators, and enzymes that can neutralize infectious agents and induce repair of damaged tissue.

Among them, MAIT cells have emerged in recent years as very important immune cells in the maintenance of homeostasis of the mucosal and non-mucosal barriers. Indeed, these cells have the specificity of recognizing specific bacterial riboflavin metabolites and display both conventional effector and tissue maintenance functions.

In this review, we first summarize the phenotypes and functions of MAIT cells in mucosal and non-mucosal barriers, then we describe their roles in these barriers and in particular their effector and tissue repair role in healthy and pathogenic conditions. Finally, we also present their associations with the other immune cells present in these barriers in the maintenance of tissue homeostasis, and the recent advances using MAIT cells in mucosal vaccination.

\section{MAIT CELL CHARACTERISTICS AT SURFACE BARRIERS}

MAIT cell localization at epithelial barriers

Although highest frequencies of MAIT cells are found in blood and liver notably in humans, ${ }^{1-4}$ they are also enriched in tissues in contact with the external environment and the commensal microbiota, including the oral mucosa, ${ }^{5}$ respiratory, ${ }^{6-8}$ intestinal, ${ }^{3,7,9-12}$ female genital tracts, ${ }^{13}$ and skin $^{14}$ (Fig. 1). In-situ analyses have shown that mucosal MAIT cells populate the basal membrane and below, and to a lesser extent the lower epithelium ${ }^{3,5,13-15}$ (Table 1). No detailed localization analysis of pulmonary MAIT cells has been conducted to our knowledge so far (Table 2). Mucosal MAIT cells therefore are situated just below the epithelium in accordance with their interaction with the commensal microbiome and their rapid-responder function to protect against immune threats and tissue injuries.

MAIT cell phenotype at epithelial barriers

MAIT cells were originally defined by co-expression of the semiinvariant TCRa chain Va7.2-Ja33/20/12 and C-type lectin-like receptor CD161 in humans in association with a limited set of $\beta$ chains, $V \beta 2, V \beta 13$, and $V \beta 22 .^{1-3,9,16,17}$ Similarly, they were defined by Va19-Ja33 expression in mice, in association with $V \beta 6$ and V $\beta 8 .{ }^{7,9,16,17}$ Almost all blood $\mathrm{Va7} .2^{+} \mathrm{CD} 161^{+} \mathrm{T}$ cells are also stained with MR1 tetramers loaded with MAIT cell agonist ligands. ${ }^{3,18}$ Such co-staining of MAIT cells has also been observed in lung fluids but remains to be investigated in other mucosal tissues. $^{18,19}$

In mucosal barriers, MAIT cell TCR composition is not well documented but is likely at least as diverse as in blood. Some studies have observed additional variability such as association of Va7.2 with Ja12, Ja27 in human gut and several TRBV chains (Table 1). In the oral human mucosa, MAIT cell TCR always includes Va7.2 but depending on the donor, the dominating Ja segment can be different (Ja33, 20, or 12$).{ }^{5}$ In contrast, blood MAIT cells from the same donors almost exclusively include Ja33 in their TCR. In mouse pulmonary mucosa, the Va19-Ja33 segment is mainly associated with $V \beta 8.1 / 8.2$ but also with $V \beta 6$ and to a lesser extent, with $\mathrm{V} \beta 2-7,8.3,9,10$, and $14 .^{7,20,21}$ Interestingly, these differences between MAIT cell TCR composition are paralleled with different responses between tissue and blood

\footnotetext{
${ }^{1}$ Université de Paris, Institut Cochin, INSERM U1016, CNRS UMR 8104, Université de Paris, Paris, France and ${ }^{2}$ Laboratoire d'Excellence Inflamex, Paris, France Correspondence: Agnès. Lehuen (agnes.lehuen@inserm.fr)

These authors contributed equally: Isabelle Nel, Léo Bertrand.
}

Received: 5 June 2020 Revised: 10 February 2021 Accepted: 12 February 2021

Published online: 22 March 2021 

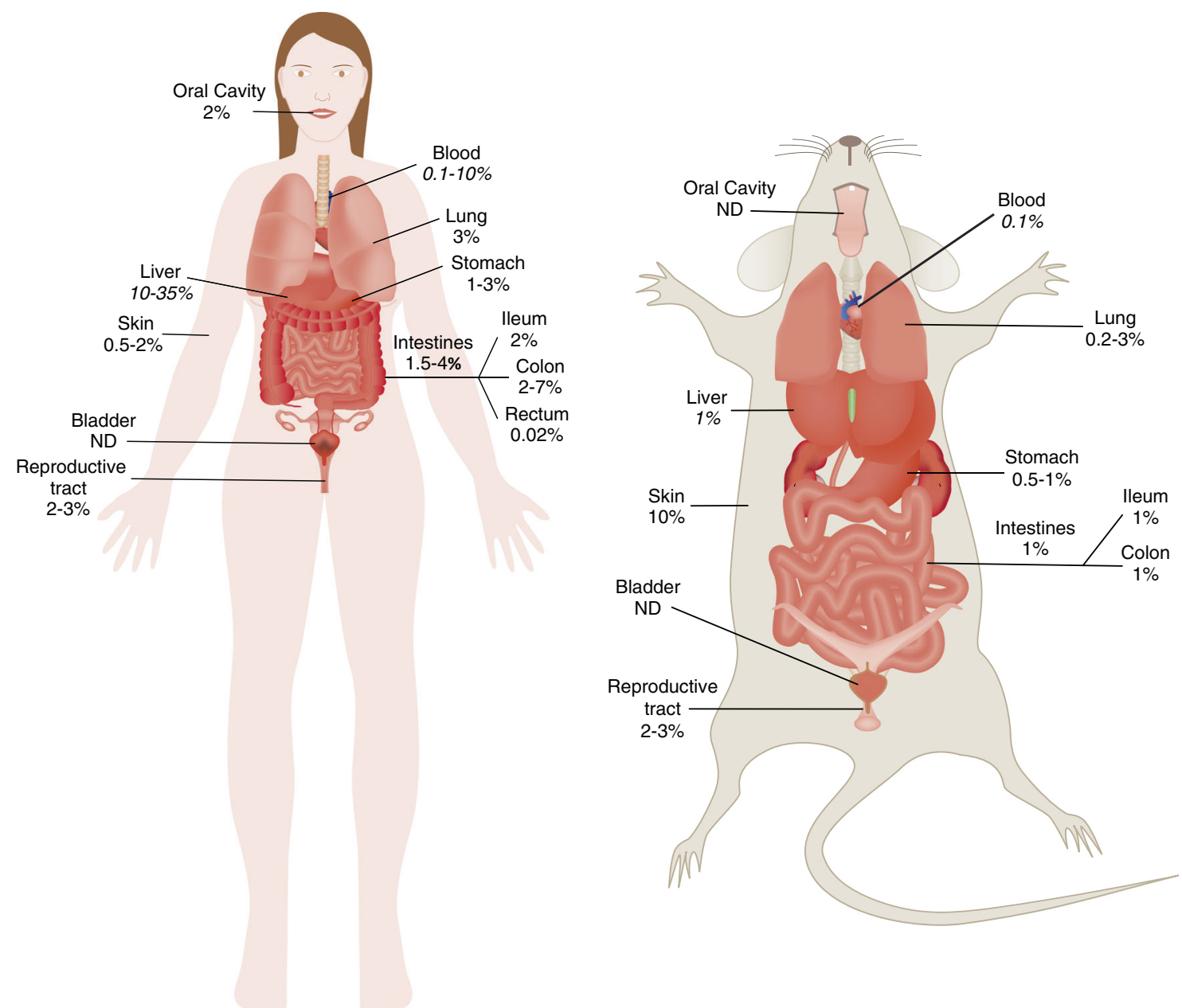

Fig. 1 Distribution of MAIT cell frequency in skin and mucosal barriers in humans and mice. Values indicate mean or range of MAIT cell frequency in the indicated tissue as \% of total T cells or $\alpha \beta T$ cells. ND: not determined; organs where MAIT cells were studied but exact frequency in humans or wild-type mice was not determined.

MAIT cells as well as distinctive transcriptomic programs in both subsets. ${ }^{5,12-14,22}$ These differences suggest therefore distinct MAIT cell functions in blood and mucosal barriers.

Expression of CD4 and CD8 on MAIT cells at epithelial barriers In humans, circulating MAIT cells are mainly $\mathrm{CD}^{+}(\sim 70 \%)$ and double negative $(\mathrm{DN})(\sim 15 \%)$ with a very low frequency of $\mathrm{CD} 4^{+}$ MAIT cells $(\sim 5 \%),{ }^{2,3,23}$ In mucosal tissues, there is a relative enrichment of DN MAIT cells that represent $\sim 40-50 \%$ of total MAIT cells, and up to $80 \%$ in some individuals. ${ }^{5,10}$ One notable exception is the female genital tract, where MAIT cell subset frequencies are similar to those observed in blood except in the endometrium where there is a slight enrichment of $\mathrm{CD}^{+}$MAIT cell frequency $\left(12 \%\right.$ of total MAIT cells). ${ }^{13}$ The high vascularization of this tract may explain this genital MAIT cell subset repartition that is closer to blood MAIT cells. Similarly in mouse skin, lung, and gut, MAIT cells are almost exclusively DN although variations can be seen depending on the strain. $7,12,14,24$

Expression of memory markers and homing receptors on MAIT cells

MAIT cells in human adult blood exhibit an effector memory phenotype $\left(\mathrm{CD}_{5} \mathrm{RO}^{+}, \mathrm{CD}_{2} 7^{+}, \mathrm{CCR} 7^{-}, \mathrm{CD} 44^{\text {high }}, \mathrm{CD} 6 \mathrm{~L}^{\text {low }}\right)$ and express numerous integrin and tissue-homing chemokine receptors (CCR5 ${ }^{\text {high }}$, CCR6 $^{\text {high }}$, CXCR6 high $^{\text {he }}$ CCR9 $\left.{ }^{\text {int }}\right) .^{2,3,23,25-27}$ This is in line with their reported or suspected increased migration toward tissues in case of local inflammation. ${ }^{2,7,8,11,12,16,22,25,26,28,29}$
Mucosal MAIT cells also share the blood MAIT cell effector memory phenotype (Tables 1 and 2). At steady state, they express markers of tissue residency $\left(\mathrm{CD}^{+} 9^{+}, \mathrm{CD}_{103}{ }^{+}\right.$, Cutaneous Lymphocyte-associated Antigen, $\mathrm{CLA}^{+}$) that can also be found on circulating MAIT cells albeit at a lower level. ${ }^{5,7,8,12,30,31}$ In the skin of healthy volunteers, up to $85 \%$ of MAIT cells can express CLA. ${ }^{32}$ It is currently unknown whether tissue-resident mucosal MAIT cells can exit mucosal tissues and recirculate. One study detected MAIT cells in the thoracic duct lymph. ${ }^{33}$ However, their exact tissue origin was not determined. There is therefore a high heterogeneity of both MAIT cell phenotypes and subsets between blood and tissues as well as between tissues themselves.

\section{Mucosal MAIT cell activation}

The semi-invariant TCR of MAIT cells is able to recognize small organic activating ligands derived from bacterial and fungal riboflavin synthesis pathways (vitamin B2) as well as nonstimulatory ligands derived from the natural photodegradation of folic acid (vitamin B9). ${ }^{34-37}$ 5-OP-RU and 5-OE-RU are, up to date, the most potent activating ligands. ${ }^{34,35}$ In addition to TCRdependent activation, they can be activated in a TCR-independent manner by several cytokines, including IL-7, IL-12, IL-15, and IL-18 (Tables 1 and 2). ${ }^{25,26,38-44}$ Currently, it has been shown that mucosal MAIT cells express several cytokine receptors, such as IL18R, IL-7R, and IL-23R. ${ }^{7,30,45-47}$

Since the commensal microbiome is the main source of riboflavin metabolites, mucosal MAIT cells are therefore likely to 
Table 1. MAIT cell phenotype in the gut mucosa during health and disease. (ND, not described).

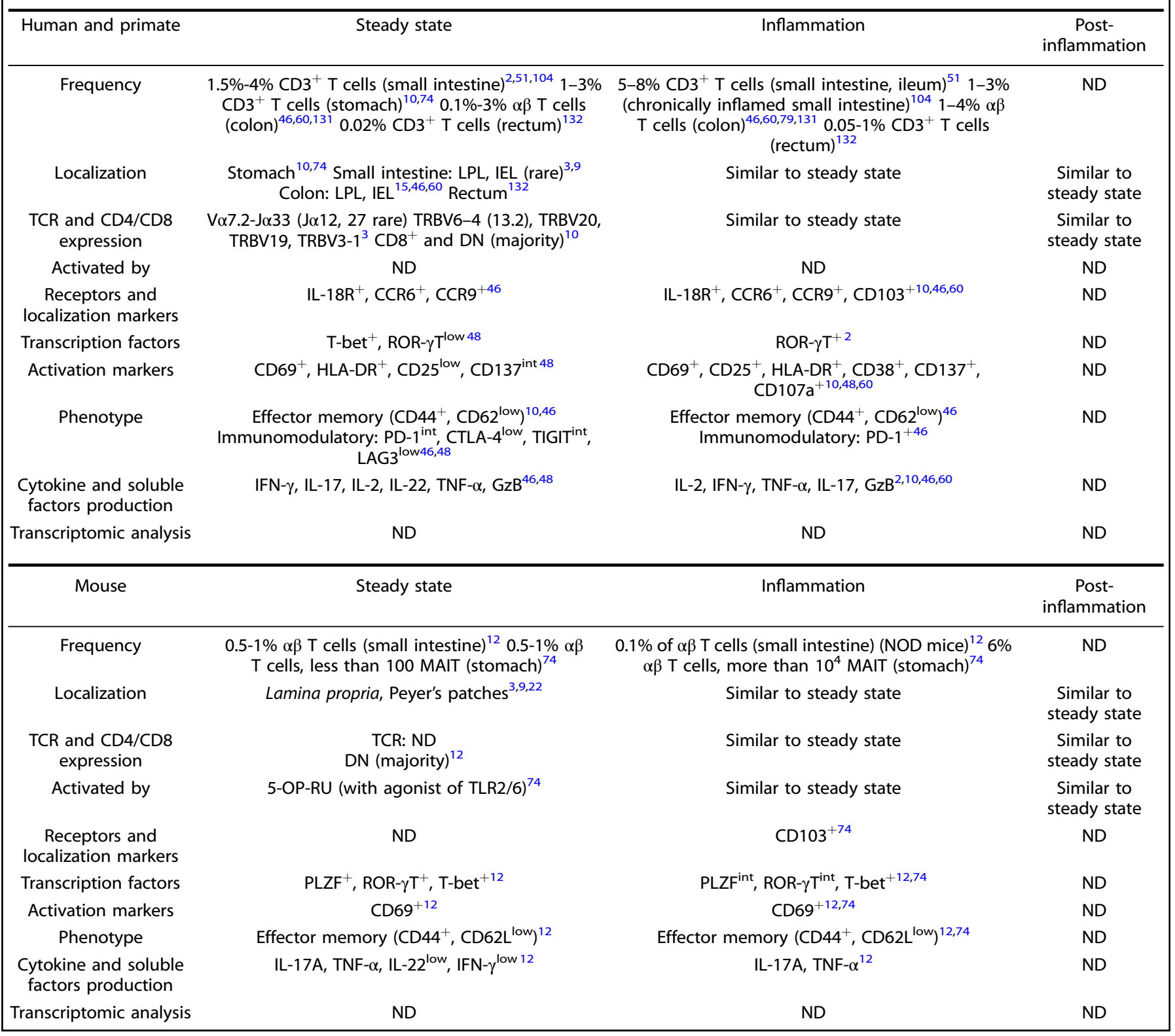

be more exposed to activating ligands, suggesting a higher, "alerted" activation state. Indeed, $\mathrm{CD}_{103}{ }^{+} \mathrm{CD} 9^{+}$tissue-resident MAIT cells express higher levels of activation markers (CD25, HLA$\mathrm{DR}, \mathrm{CD} 38)$ compared to passing-through $\mathrm{CD}_{103}^{-} \mathrm{CD}^{-} 9^{-}$ MAIT cells. ${ }^{5,31}$ In the human gut, MAIT cells can express immunomodulatory receptors (TIGIT, PD-1, LAG-3, CTLA-4). ${ }^{46,48}$ PD $-1^{+}$MAIT cell frequency is very high at steady state and can reach up to $80 \%$ of mucosal MAIT cells in some individuals. This suggests mechanisms of immune checkpoints to limit mucosal MAIT cell activation at steady state.

Mucosal MAIT cell cytokine profile at barriers

In humans as in mice, activated MAIT cells can produce a broad range of cytokines including Th1-type (TNF- $a$, IFN- $\gamma$, IL-2), Th2-type (IL-4, IL-10), and Th17-type cytokines (IL-17, IL-22). ${ }^{2,7,26}$ Analysis of MAIT cells isolated from different barriers and stimulated with PMA and ionomycin suggests distinct profiles of polarization depending on the tissue of origin. In mice, MAIT cells from the gut and skin preferentially produce IL-17 to the detriment of IFN- ${ }^{12,14}$
This polarization is also observed in human oral mucosa compared to circulating MAIT cells, in association with a tendency toward increased granzyme $\mathrm{B}^{+}$(GzB) MAIT cell frequency. ${ }^{5}$ MAIT cells isolated from the human female genital tract and stimulated in vitro by fixed Escherichia coli for $24 \mathrm{~h}$ produce more IL-17 and IL-22 than blood MAIT cells that produce more IFN- $\gamma$, TNF- $a$, and $\mathrm{GzB} .{ }^{13}$ Even though mucosal MAIT cells seem to be skewed towards IL-17 and IL-22 production at steady state, further studies are still required to investigate more precisely the likely specific roles of MAIT cells in the various mucosal barriers.

\section{ROLES OF MAIT CELLS AT MUCOSAL BARRIER SITES}

Autonomous abilities of MAIT cells

MAIT cell frequency increases in infected and inflamed tissues, either through migration from blood $8,11,12,26,28,29$ or local proliferation at infected sites, ${ }^{30,49,50}$ although the relative importance of both phenomena seems to depend on the encountered pathogen as well as the infected tissue. Once in the tissue, 
Table 2. MAIT cell phenotype in the pulmonary mucosa during health and disease. (ND, not described).

\begin{tabular}{|c|c|c|c|}
\hline Human and primate & Steady state & Inflammation & Post-inflammation \\
\hline Frequency & $\begin{array}{l}0.2 \% \text { of } \mathrm{CD}^{+}{\text {cells }(\mathrm{BALF})^{31}}^{31} \% \alpha \beta \\
\mathrm{CD}^{+} \mathrm{T}^{+} \text {cells }^{30}\end{array}$ & $0.5-10 \%$ of $\mathrm{CD}^{+}$cells $(\mathrm{BALF})^{19,31}$ & ND \\
\hline $\begin{array}{l}\text { TCR and CD4/CD8 } \\
\text { expression }\end{array}$ & $\begin{array}{c}V \alpha 7.2^{45} \\
\text { CD4/CD8 expression: ND }\end{array}$ & Similar to steady state & Similar to steady state \\
\hline Activated by & 5-OP-RU, IL-2, IL-12, IL-7, IL-18 & Similar to steady state & Similar to steady state \\
\hline $\begin{array}{l}\text { Receptors and } \\
\text { localization markers }\end{array}$ & $\mathrm{CD} 5^{+}, \mathrm{IL}-18 \mathrm{R}^{+30,45}$ & $\mathrm{CD}_{103}{ }^{+}, \mathrm{IL}^{-18 \mathrm{R}^{+}}, \mathrm{CCR}^{+31,45}$ & ND \\
\hline Transcription factors & ND & ND & ND \\
\hline Activation markers & $\mathrm{CD} 69^{+30}$ & $\mathrm{CD} 69^{+}, \mathrm{CD} 25^{+}, \mathrm{CD} 107 \mathrm{a}^{+31,41,58,59,133}$ & ND \\
\hline Phenotype & $\begin{array}{c}\text { Memory }\left(\mathrm{CD} 45 \mathrm{RO}^{+}, \mathrm{CCR7}^{-}, \mathrm{CD}^{\text {low }} \mathrm{L}^{\text {low }}\right) \\
\mathrm{Ki}-67^{\text {low }}\end{array}$ & $\begin{array}{c}\text { Memory }\left(\mathrm{CD} 45 \mathrm{RO}^{+}, \mathrm{CCR7}^{-}, \mathrm{CD}^{\prime} \mathrm{L}^{\text {low }}\right) \\
\mathrm{Ki}-67^{+30,58}\end{array}$ & ND \\
\hline $\begin{array}{l}\text { Cytokine and soluble } \\
\text { factors production }\end{array}$ & ND & GzB, TNF, GM-CSF, IL-17/IFN- $\gamma^{6,30,31,41,58,59}$ & GM-CSF, IL-17/IFN- $\gamma^{58}$ \\
\hline $\begin{array}{l}\text { Transcriptomic } \\
\text { analysis }\end{array}$ & ND & $\begin{array}{c}\text { Th17 genes: IL17A, CSF2, IL-21, IL-32, HIF1A, } \\
\text { AHR, BATF, STAT3 IL23R, IL17RA, RORC, RORA, } \\
\text { LTB, GPR65 Th1 genes: IFNG, TBX21, STAT4, } \\
\text { CD8B Innateness markers: ZBTB16/PLZF, NKG7, } \\
\text { KLGR1, NCR3, HOPX }{ }^{31}\end{array}$ & ND \\
\hline Mouse & Steady state & Inflammation & Post-inflammation \\
\hline Frequency & $0.4-3 \% \alpha \beta$ T cells ${ }^{7,21,29}$ & $2 \%-60 \% \alpha \beta$ T cells $8,20,21,29,43,49,74$ & $10-20 \% \alpha \beta$ T cells ${ }^{8,49}$ \\
\hline $\begin{array}{l}\text { TCR and } C D 4 / C D 8 \\
\text { expression }\end{array}$ & $\begin{array}{c}\text { V } \alpha 19-J \alpha 33 \text { V } \beta 8.1 / V \beta 8.2 \text { (majority) V } \beta 6 \\
\text { V } \beta 2-7,8.3,9,10,14,20,21 \\
\text { DN (majority) }{ }^{24}\end{array}$ & Similar to steady state & Similar to steady state \\
\hline Activated by & $\begin{array}{l}\text { 5-OP-RU (with agonists of TLR2/6, } \\
\text { TLR3, TLR4, TLR9) IL-12, IL-15, IL-7, IL- } \\
\text { 18, IL-23 (expansion) }{ }^{20,21,43,44,47}\end{array}$ & Similar to steady state & Similar to steady state \\
\hline $\begin{array}{l}\text { Receptors and } \\
\text { localization markers }\end{array}$ & 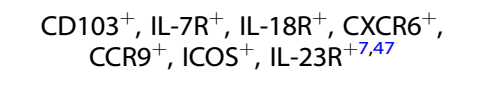 & 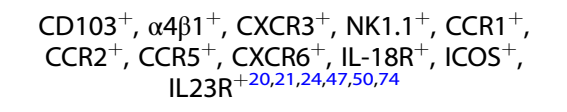 & 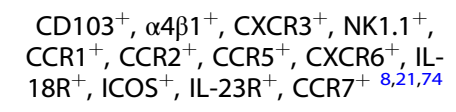 \\
\hline Transcription factors & $\begin{array}{c}\text { PLZF }^{+} \text {(except CD4 }{ }^{+} \text {MAIT), ROR- } \gamma T^{+} \\
\text {Tbet }^{\text {low } 7,47,49}\end{array}$ & $\mathrm{PLZF}^{+}, \mathrm{ROR}^{-} \gamma \mathrm{T} / \mathrm{T}-\mathrm{bet}^{+8,47,49}$ & $\mathrm{PLZF}^{+}, \mathrm{ROR}-\gamma \mathrm{T} / \mathrm{T}-$ bet $^{+8,49}$ \\
\hline Activation markers & $\mathrm{CD} 69^{-/ \mathrm{low} 7,8}$ & $\mathrm{CD} 69^{+}, \mathrm{CD} 25^{+}, \mathrm{CD} 107 \mathrm{a}^{+8,43}$ & $\mathrm{CD} 69^{+8}$ \\
\hline Phenotype & 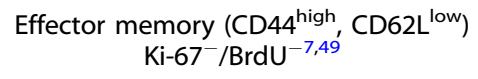 & $\begin{array}{l}\left.\text { Effector memory (CD44 }{ }^{\text {high }}, \mathrm{CCR7}^{-}, \mathrm{CD}^{\prime} \mathrm{L}^{\text {low }}\right) \\
\mathrm{Ki}-67^{+} / \mathrm{BrdU}^{+8,21,49,50}\end{array}$ & 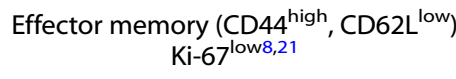 \\
\hline $\begin{array}{l}\text { Cytokine and soluble } \\
\text { factors production }\end{array}$ & IL-17 (low) ${ }^{7}$ & $\begin{array}{c}\text { GM-CSF, IL-2, IL-17, IFN- } \gamma \text {, LIF, TNF, iNOS, GzB, } \\
\text { GzK (high) })^{8,20,21,24,43,49,64} \text { LTA, M-CSF } \\
\text { (moderate) }{ }^{21} \text { CXCL1, CCL3, CCL4, CXCL16 }{ }^{21}\end{array}$ & $\begin{array}{l}\text { GM-CSF, IL-17/IFN- } \gamma \text {, TNF (high to } \\
\text { moderate) } \\
\text { CCL3, CCL4, CXCL16 } 16^{21}\end{array}$ \\
\hline $\begin{array}{l}\text { Transcriptomic } \\
\text { analysis }\end{array}$ & $\begin{array}{l}\text { Tissue localization }{ }^{22} \text { TCR activation } \\
\text { (Jun, Fos, Junb, Nfkbie, Nr4a1) })^{22}\end{array}$ & $\begin{array}{l}\text { Tissue repair and angiogenesis genes (Tnf, } \\
\text { Csf2, Hif1a, Furin, Vegfb, Ptges2, Pdgfb, Tgfb1, } \\
\text { Mmp25, Hmgb1)14,15,21,56 Cell cycle and } \\
\text { signaling pathways (MKi-67, Kif18) } \\
\text { Immunoregulatory (Rankl, Trance) })^{21}\end{array}$ & $\begin{array}{l}\text { Immunomodulatory and memory- } \\
\text { promoting genes (Gitrl, II-15, Rankl, } \\
\text { Trance })^{21}\end{array}$ \\
\hline
\end{tabular}

activated pro-inflammatory mucosal MAIT cells produce both cytokines and cytotoxic mediators. TNF- $a$, IL-17, and IFN- $\gamma$, the three main cytokines produced by MAIT cells, exert their effects mainly through stimulation of other actors of the immune system. $2,5,13,25,26,31,51$ TNF- $a$ and IFN- $\gamma$ also independently help to suppress pathogens. Indeed, both can directly inhibit viral replication in infected cells and induce growth arrest and apoptosis of cancerous cells. ${ }^{52-54}$

Different cytotoxic mediators also allow MAIT cells to directly kill target cells. At steady state, MAIT cells possess low cytotoxicity. ${ }^{55,56}$ They can produce granzyme $A(\mathrm{GzA})$ and $\mathrm{K}$ (GzK) but have low levels of GzB and perforin expression. ${ }^{55,56}$ TCRdependent activation increases $\mathrm{GzB}$ and GzM production and reduces GzA and GzK production, while dual TCR-dependent and independent stimulation increases expression of all types of granzymes. ${ }^{55,56}$ MAIT cells also express perforin and granulysin at high levels after TCR-dependent and independent stimulation. ${ }^{55,56}$
Interestingly MAIT cells can also express the FasL/sFasL death ligands, although the relative amount of cytotoxicity performed through this pathway remains to be quantified precisely. ${ }^{56}$

Increased CD107a expression confirms that MAIT cells kill their targets by degranulating cytotoxic granules after TCR-MR1 engagement, including bacteria-infected epithelial cells as well as cancerous cells. ${ }^{57-60}$ MAIT cells can notably exert their cytotoxicity against epithelial cells infected by E. coli, ${ }^{55}$ Shigella flexneri, ${ }^{57}$ or macrophages infected by Helicobacter pylori, ${ }^{10}$ and on leukemic cancerous cells. ${ }^{60}$ Direct cytotoxic effects of MAIT cells against virus-infected cells have also been suggested but the exact mechanism remains to be fully demonstrated. ${ }^{18,41-43}$ Of note, these detailed studies of the kinetics and specificities of MAIT cell cytotoxicity were performed in in vitro activation settings on blood MAIT cells, and their complete transposition to mucosal MAIT cells remains to be confirmed. 
MAIT cells against mucosal and epithelial barrier threats

The abilities of MAIT cells to recognize bacterial metabolites in a pro-inflammatory context initially suggested their key role to fight bacterial and fungal infections. ${ }^{20,45,47,49,61-65}$ Both TCRdependent ${ }^{6,8,10,45,49}$ and TCR-independent ${ }^{8,20,38,62,66}$ activation of MAIT cells have been reported during bacterial infection. MAIT cells are therefore also involved in immune responses against bacteria that do not synthesize MAIT cell-activating ligands. In both humans and mice, circulating MAIT cell frequency inversely correlates with disease severity, ${ }^{6,28,58,67,68}$ bacterial load or susceptibility to develop bacterial infections. ${ }^{69}$ Mouse models of bacterial infection lacking MAIT cells show increased bacterial load (E. coli, Mycobacterium bovis BCG, Mycobacteria abscessus, Klebsiella pneumoniae, Francisella tularensis, Legionella longbeachae). ${ }^{20,45,47,49,61,62}$ MAIT cells were also sufficient to ensure survival upon infection with $L$. longbeachae in otherwise severely immunocompromised $\operatorname{Rag}^{-1-} \mathrm{Yc}^{-}$mice. ${ }^{49}$

However, recent studies have surprisingly shown no critical involvement of MAIT cells to clear Mycobacterium tuberculosis infection in mice. ${ }^{29,70,71}$ Along the same line, a recent case report of a patient lacking MAIT cells showed mild persistent viral infections but not increased bacterial infections. ${ }^{72}$ Of note, this patient presented a notable expansion of $\mathrm{V} \delta 2^{+} \gamma \delta \mathrm{T}$ cells that may have compensated for some of the functions normally performed by MAIT cells. Moreover, some studies reported a potentially deleterious role of MAIT cells in bacterial infections. ${ }^{73,74}$ Indeed, $\mathrm{Mr}^{-1-}$ mice were less sensitive to Clostridium difficile infections. ${ }^{73}$ Meanwhile, $\mathrm{Mr}^{-1-}$ mice were less impacted by chronic infection of the gastric mucosa by $H$. pylori as here, MAIT cells fueled immune-mediated bystander damage to the mucosa. ${ }^{74}$ Therefore, the exact direct contribution of MAIT cells in the clearance of bacterial pathogens probably depends on the nature of the pathogen itself, and its ability to stimulate a MAIT cell proinflammatory response, notably through its production of MAIT cell-activating ligands and the production of MAIT cellactivating cytokines by the local immune system. ${ }^{29}$

Viruses can also induce MAIT cell activation. ${ }^{42,75}$ Most of our knowledge on the interactions between MAIT cells and viral infections in the mucosa derives from the study of Human Immunodeficiency Virus 1 (HIV-1), ${ }^{75-79}$ Influenza A virus (IAV) ${ }^{41-43,75}$ and recently Severe Acute Respiratory Syndrome CoronaVirus 2 (SARS-CoV-2) infections. ${ }^{18,19,80}$ Colon and rectum MAIT cell frequencies remain similar in HIV-1-positive patients compared to uninfected controls, ${ }^{76,77,79}$ although one study reported diminished levels. ${ }^{78}$ During IAV infection, $\mathrm{Mr}^{-1-}$ mice had a greater weight loss and mortality rate compared to control mice while exogenous transfer of MAIT cells improved both parameters. ${ }^{43} \mathrm{Mr}^{-1-}$ mice had reduced numbers of alveolar macrophages and pulmonary $T$ cells, as well as accumulation of conventional antigen-specific $T$ cells. Pulmonary damage, described as total proteins in the broncho-alveolar lavage fluid, was also increased in $\mathrm{Mr}^{-1-}$ mice. Conversely, transfer of MAIT cells significantly delayed fatal outcome through IFN- $\gamma$ production upon infection by IAV in otherwise severely immunocompromised Rag2 ${ }^{-1-} \gamma \mathrm{c}^{-}$mice. ${ }^{43}$ Moreover, we and other groups recently unveiled alterations of MAIT cells during SARS-CoV-2 infection. ${ }^{18,19,80}$ Blood MAIT cells were significantly reduced in frequency, highly activated (high CD69, CD56, IFN- $\gamma$, and GzB expression), and those parameters were linked with pulmonary function loss, global inflammation levels, and fatal outcome of severe patients. Activation levels of MAIT cells correlated with those of innate cell subsets, NK, ILC, and $\gamma \delta T$ cells that play significant roles in mucosa immunity. However, MAIT cell activation was significantly superior. ${ }^{18}$ MAIT cell frequency was increased in infected lungs and they also displayed higher levels of CD69, GzB, and PD-1 compared to blood MAIT cells. ${ }^{18,19}$ Finally, SARS-CoV-2-infected macrophages were able to activate MAIT cells in an IFN-a/IL-18 dependent manner. Therefore, compared to IAV infection, MAIT cells seem to have an ambivalent role in SARS-CoV-2 infection..$^{18,19,41-43,75,80}$ In an early phase, MAIT cells seem to have a protective role, ${ }^{19}$ however, their sustained activation could participate in pulmonary lesions. ${ }^{18,80}$ Of note, iNKT cells that share many functional similarities with MAIT cells displayed a close phenotype with those cells during SARS-CoV-2 infection. ${ }^{19}$

Interestingly, recent publications highlighted that mucosal MAIT cells could be targeted by viral infections. Rectum CD4 ${ }^{+}$ MAIT cells were preferentially lost during HIV-1 progression likely due to direct infection by HIV- $1,{ }^{77}$ while CD8 ${ }^{+}$MAIT cells appeared to be resistant to infection. ${ }^{76}$ Blood MAIT cells were infected and programmed for apoptosis by measles, as they expressed the CD150 entry receptor for the virus. ${ }^{81}$ The receptivity of their mucosal counterparts remains unknown. Herpes Simplex Virus-1 (HSV-1) blocked MR1 upregulation in acute infected cells and therefore might hamper MAIT cell TCR-MR1 activation. ${ }^{82}$ Since HSV-1 latent infection is widespread in the general population in oral and genital mucosa, it would be interesting to explore if this repression is operating in this context and its impact on the MAIT cell-microbiota interaction.

Another category of viruses susceptible to affect MAIT cellmicrobiota interactions are bacteriophages. In addition to their direct effects on the microbiota, ${ }^{83}$ several studies have also suggested that bacteriophages might be able to activate or suppress the innate immune system through the DNA-detecting Toll-like receptors (TLR), and therefore might perhaps affect MAIT cells as well. ${ }^{84}$ As bacteriophages, bacteria and host constitute a unique, interacting ecosystem, the interplay between MAIT cells, bacteriophages and bacteria might be a promising research field to explore in the future. Overall, upon bacterial and viral infections, MAIT cells play an ambivalent role depending on the pathogen and the phase of infection.

Likewise, knowledge on MAIT cell involvement against mucosal cancers remains incomplete. Several studies have reported increased MAIT cell frequencies in mucosal tumors compared to healthy surrounding tissue in colorectal, gastric, lung and esophageal cancers and suggested recruitment of blood MAIT cell through CCL20-CCR6 chemotaxis. ${ }^{46,60,85-87}$ However, no clear consensus has emerged as to whether they mainly display pro- or anti-tumor properties. Indeed, tumor-infiltrating MAIT cells were shown to produce IFN- $\gamma$, cytotoxic mediators, and mounted Th1 responses. ${ }^{46,85,88}$ MAIT cells were also able to reduce viability of an esophageal cancer line as well as interrupting cell division of colon cancer cells in an MR1-dependent manner. ${ }^{46,86,89}$ However, several studies reported that MAIT cell IFN- $\gamma$ production was suppressed when exposed to mucosal cancer cells or their culture medium, suggesting MAIT cell immune suppression by tumoral cells. ${ }^{46,85,90}$ Worse, MAIT cells could be actively pro-tumorigenic cells. Accumulation of MAIT cells in colorectal cancer lesions was associated with less favorable outcome. ${ }^{87} \mathrm{Mr}^{-1-}$ mice significantly developed fewer metastases from skin melanoma and protection was reversed after infusion of MAIT cells. ${ }^{91}$ Yan and colleagues demonstrated that MAIT cells could be activated by MR1-expressing mucosal cancer cells, including melanomas and adenocarcinomas. Yet, strikingly, activated MAIT cells inhibited NK cell activity by producing IL-17. Moreover, treatment with the MAIT cell inhibitory ligand acetyl-6-formylpterin or anti-MR1 antibodies resulted in significant tumor growth reduction. Therefore, it is likely that protective or deleterious functions of MAIT cells against mucosal cancers heavily depend on whether they adopt a Th17 or Th1 profile. Future studies would be of interest to fully understand their actions in these situations.

Mucosal barrier integrity maintenance and repair by MAIT cells In addition to preventing any immune threats, the mucosal immune system is heavily involved in the maintenance of mucosal tissues, ${ }^{92}$ and recent advances have shown MAIT cell involvement as well. ${ }^{12,14,15,21,56}$ We first proposed that MAIT cells protect gut integrity via the production of IL-17 and IL-22, ${ }^{12}$ and another study 
showed similar cytokine productions in the genital mucosa as well. $^{13}$ In the gut, production of IL-22 promotes epithelial cell survival and proliferation ${ }^{93}$ and mucus production by goblet cells $^{94}$ while IL-17 participates in the regulation of Occludin to prevent excessive barrier permeability during epithelial injury. ${ }^{95}$ Accordingly, IL-17A produced by MAIT cells strengthened the colonic mucosal barrier and prevented local activation of proinflammatory Th1 and Th17 conventional T cells. ${ }^{96}$

Recently, several groups discovered almost simultaneously a new function of MAIT cells in tissue repair. Transcriptomic studies on human circulating TCR-activated MAIT cells, pulmonary MAIT cells from mice acutely infected with $L$. longbeachae and murine skin MAIT cells unveiled expression of shared tissue protection and repair genes. ${ }^{14,15,21,56}$ It included, among others (full list can be found in ${ }^{14,15,21,56}$ ), immune genes involved in tissue repair (TNF, PTGES2, TGFB1, CCL3, HMGB1), proteases (Furin, MMP25), growth factors (GM-CSF, M-CSF, PDGFB, LIF) as well as angiogenic genes (HIF1A, VEGFB). This tissue repair transcriptome was similar to the transcriptome of murine $\mathrm{H} 2-\mathrm{M} 3$ restricted commensal-specific Tc17 that are central in skin repair, ${ }^{97}$ and required TCR-dependent activation of MAIT cells for transcription. ${ }^{15,21,56}$ Expression at protein level was confirmed for TNF, Furin, CCL3, and GM-CSF after TCR-specific activation of human circulating $\mathrm{CD} 8^{+}$MAIT cells. ${ }^{15}$

Moreover, human circulating $\mathrm{CD}^{+}$MAIT cells activated by $E$. coli supernatants accelerated wound closure in an in vitro woundhealing assay utilizing intestinal epithelial cells, in an MR1dependent manner. ${ }^{15}$ Direct 5-OP-RU application in vivo on wounded skin was sufficient to also expand MAIT cells and accelerate tissue repair in mice. ${ }^{14}$ However, direct evidence of MAIT cell tissue repair function in humans remains to be described. Interestingly, the tissue repair ability of MAIT cells seems to be direct but could also be mediated through production of chemokines (CCL3) that recruit other tissue-repair immune cells, such as macrophages.

\section{MAIT CELLS, PROTECTIVE OR PATHOLOGICAL?}

MAIT cell dysfunction in mucosal diseases

Both physiological functions of MAIT cells, mucosa maintenance and protection against pathogens, may be deleterious either by having insufficient or exacerbated effects.

As described earlier, the majority of $\mathrm{Mr}^{-1-}$ mouse models of infections present higher pathogen loads and reduced survival upon pathogen challenge, pointing to a conserved protective role of MAIT cells that was impaired when they were diminished. ${ }^{20,26,43,45,47,49,61,62}$ In Type 1 diabetes (T1D) the loss of IL-17 and IL-22 production by gut MAIT cells weakened mucosal barrier integrity. ${ }^{12,95,98-100}$ This increase of gut permeability resulted in local gut inflammation and translocation of bacterial compounds that may reach the liver or pancreatic lymph nodes, exacerbating local autoimmunity and disease. ${ }^{12,98}$ This loss of IL-17 and IL-22 was induced by autoreactive $T$ cells localizing into the gut, rather than hyperglycemia per se. Interestingly, the only HIV-1 study reporting diminished levels of MAIT cells in the gut also reported increased bacterial DNA in the blood, supporting links between impaired gut integrity and loss of MAIT cell functionality. ${ }^{78}$

The same loss of mucosal integrity may also, on the contrary, be the result of inflammatory cytokine overproduction by MAIT cells as observed in Type 2 diabetes (T2D), obesity, and metabolic diseases. ${ }^{11,26,101,102}$ When fed a high fat diet, MAIT cells in the ileum of $\mathrm{C57BL} / 6 \mathrm{~J}$ wild-type mice produced elevated levels of IL-17 compared to normal diet-fed mice. Moreover, obese Va19Ja33-TCR transgenic mice (with tenfold more MAIT cells than wildtype mice) displayed elevated mRNA transcription of inflammatory genes such as $I 117$ or Tnfa and a decreased expression of antiinflammatory genes $(1 / 5,1 / 13)$ in ileum as compared to nontransgenic littermate mice. This exacerbated inflammation was associated with decreased expression of tight junction genes (Tjp1, Ocln, Cldn4) associated with increased gut permeability. Conversely, $\mathrm{Mr}^{-1-}$ mice fed with the same high fat diet were protected against ileum inflammation and metabolic disorders. ${ }^{101}$

MAIT cells might also participate in the pathogenesis of human intestinal chronic inflammatory diseases, including Crohn's disease and ulcerative colitis. ${ }^{51,103,104}$ Two studies reported increased MAIT cell frequency in chronically inflamed gut tissue ${ }^{51,103}$ while another reported a decrease of those cells. ${ }^{104}$ Loss of MAIT cells may weaken the gut barrier resulting in high inflammation because of gut breaches, ${ }^{104}$ or on the contrary high IL-17 production from MAIT cells might fuel chronic inflammation. ${ }^{51}$

In the lungs, as in the gut, high expression of $\mathrm{IL}-17$ can be deleterious for epithelial barrier integrity. ${ }^{105,106}$ Increase of IL-17/ IFN- $\gamma$ ratio production from MAIT cells tended to associate with aggravated community-acquired pneumonia. ${ }^{31}$ MAIT cells may also have deleterious effects in pulmonary cancer. In mice, IL-17 produced by MAIT cells inhibited NK cell antimetastatic response, ${ }^{91}$ and increased blood vessel permeability, E-selectin and VCAM-1 expression by lung endothelial cells, mechanisms that could all be involved in the dissemination of metastases. ${ }^{107}$

Finally, cytotoxic MAIT cells may exert direct harmful effects in the mucosae. In the context of SARS-CoV-2 infection, we found high levels of GzB production by lung MAIT cells as well as high GzB and CD107a expression by MAIT cells cocultured with infected SARS-CoV-2 macrophages, suggesting that MAIT cells could kill virus-infected cells. ${ }^{18}$ MAIT cells might also contribute to tissue damage by killing non-infected neighboring cells that have upregulated MR1 under inflammation., 12,18,19,101 Along the same line in oral lichen planus, invasion of oral epithelial cells by Candida spp. could lead to an MR1-dependent cytotoxic response of MAIT cells. This response may participate in the loss of oral mucosal integrity, a hallmark of this pathology. ${ }^{108}$

Finally, it is also possible that dysregulation of mucosal MAIT cell tissue repair ability might participate in the development of fibrosis ${ }^{109}$ as observed in the liver with the enhancement of the profibrogenic function of myofibroblasts by MAIT cells., ${ }^{410}$

How does the MAIT cell response vary according to the context? Translocation of bacterial components, especially in the gut, occurs even within a healthy mucosal barrier. ${ }^{111}$ The microbiome is essential for MAIT cell development, ${ }^{9}$ and strong evidence now shows that it is key in modulating MAIT cell activation.

Activation of mucosal MAIT cell depends on the availability of activating ligands that are produced by the local microbiota. Bacterial species of the Bacteroidetes and Proteobacteria phyla had statistically higher MAIT-TCR stimulation capacity compared to Firmicutes or Actinobacteria. ${ }^{112}$ Activation of MAIT cells was correlated with amounts of riboflavin production from bacteria. Another study performed with an in vitro engineered human microbiota confirmed that only the MAIT cell activating bacterial strains Beta thetaiotaomicron, E. coli, and Lactobacillus plantarum had all enzymes for riboflavin biosynthesis among the eight species composing this artificial microbiota. ${ }^{113}$ Microbiome diversity and stress conditions can also influence MAIT cell activating ligand availability. A diverse microbiota seemed to consume more riboflavin. ${ }^{113}$ Under acid stress, this riboflavin consumption was further increased resulting in lower MAIT cell activation. ${ }^{113}$ Similarly, we demonstrated that altered microbiota in obese mice produced less MAIT cell activating ligand. ${ }^{101}$ This suggests that a stressed, unbalanced microbiota potentially results in a diminished TCR-dependent MAIT cell activation. Carbohydrate sources and oxygen availability also modulate riboflavin production from microbiota. ${ }^{48}$ However, the exact implications of these modulations of riboflavin production from the microbiome on MAIT cell activation remain to be fully elucidated. 


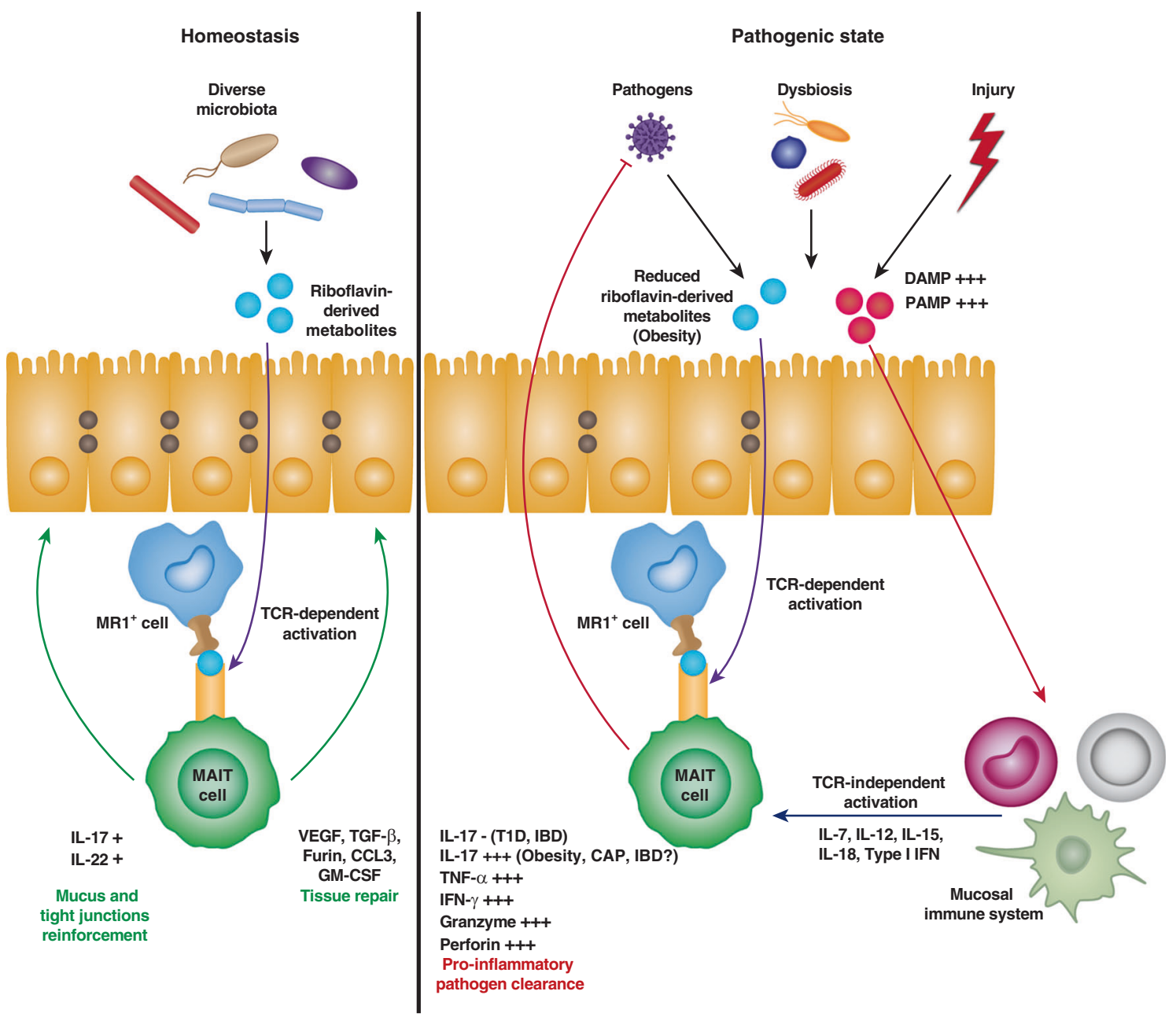

Fig. 2 MAIT cell functions depend on activation pathways and inflammatory context at barrier sites. At homeostasis (left panel), activated MAIT cells act as guardians of barrier integrity by producing IL-17 and IL-22 and expressing several tissue repair factors. In this context MAIT cells might be activated by ligands provided by commensal bacteria. In a pathogenic context (infection by pathogens, dysbiosis or injury) (right panel), immune stimulatory molecules increase at barrier sites, resulting in a concomitant TCR-dependent and TCR-independent activation of MAIT cells. In this context, MAIT cells produce high levels of pro-inflammatory cytokines, cytotoxic mediators, and contribute to pathogen clearance. Yet excessive pro-inflammatory activation of MAIT cells might also aggravate chronic mucosal inflammatory diseases.

Activation of MAIT cells also critically depends on the presence of co-activating signals from the local innate immune system, suggesting a two-signal model ${ }^{114}$ as described for conventional T cell activation. ${ }^{115}$ Thus, TCR-dependent activation is not sufficient to induce full effector function (IFN- $\gamma$, TNF- $\alpha$, and cytotoxic mediator production) and may only promote tissue repair by MAIT cells. ${ }^{14,15,21}$ Indeed, several groups have demonstrated that acquisition of high cytokine production and especially granzyme and perforin production by $\mathrm{CD}^{+}$MAIT cells is dependent on a combination of TCR-dependent and independent signals (IL-7, IL12, IL-15, IL-18, Type I IFN, or CD28/CD2 costimulatory molecules). ${ }^{15,39,55,56,116}$

At steady state, when there is a diverse microbiota, mucosal MAIT cells may present a basal activity, characterized by TCRdependent activation that promotes tissue repair and mucosa barrier through the production of IL-17 and IL-22. However, when there are either microbiota alterations, pathogen invasion or mucosal barrier breaches, pro-inflammatory signals issued by the local immune system lead to TCR-independent activation of MAIT cells in addition to TCR-dependent activation. This switches MAIT cells toward a pro-inflammatory setting with high production of IFN- $\gamma$, TNF- $\alpha$ in conjunction with cytotoxic abilities. IL-17 production however is lost in T1D in gut, but increased in T2D in gut and in community-acquired pneumonia (CAP) in lungs. Therefore, MAIT cells help to suppress any microbial threat but they can also fuel debilitating mucosal inflammation (Fig. 2).

\section{MAIT CELL INTERACTIONS WITH OTHER CELLS OF THE MUCOSA}

MAIT cells are in interaction with several other immune and nonimmune cells in mucosal tissues, on which they both depend and act on during inflammation and exposure to dangers.

MAIT cell activation by immune cells

MAIT cells can be activated via "professional" APCs, mainly dendritic cells (DCs), macrophages, and monocytes, depending on the nature of the pathogen (Fig. 3). Activation of MAIT cells can be mediated through presentation of riboflavin metabolites on MR1 by these cells, notably in M. tuberculosis, $H$. pylori, Streptococcus pneumoniae, Salmonella enterica Typhimurium, and L. longbeachae infections. ${ }^{8,45,47,49,74,117}$ TLR stimulation of professional APCs both increases their MR1 surface expression but also induces production of cytokines able to modulate MAIT cell activation, effector potential, and proliferation. ${ }^{21,116,118}$ IL-7, IL-12, IL-15, and IL-18 can directly activate MAIT cells in context of IAV, 


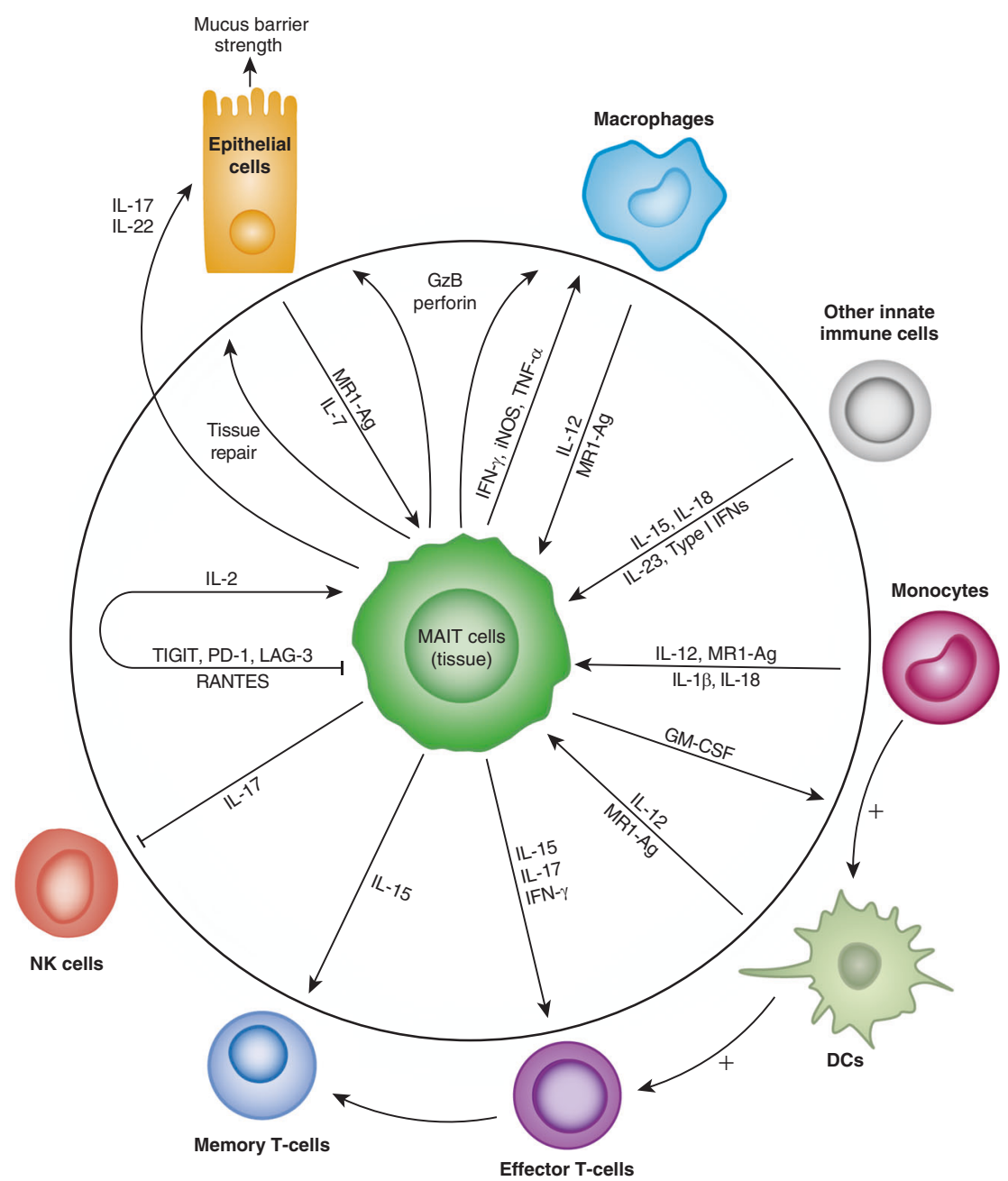

Fig. 3 MAIT cells are at the crossroad of the epithelial barrier immune system. At barrier sites, MAIT cells play a direct role in barrier integrity but also strongly interact with actors of both innate and adaptive immune systems. Innate immune cells and epithelial cells can provide activation signals to MAIT cells, through cytokines or MR1-dependent pathways. After activation and depending on the context, MAIT cells can modulate their activity and sustain barrier immunity through cytokines and tissue repair. They also foster all stages of the immune responses by boosting activation at the early phase of macrophages with TNF- $\alpha$, iNOS, and IFN- $\gamma$, activation of dendritic cells with GM-CSF, of effector lymphocytes with IL-15, 17, and IFN- $\gamma$ and finally, of memory cells with IL-15.

SARS-CoV-2, and S. pneumoniae infections. ${ }^{18,38,40-44,66,75}$ Type I IFNs, which can be produced by plasmacytoid dendritic cells after TLR7 stimulation, and IL-7 are both able to enhance MAIT cell cytotoxic abilities. ${ }^{18,39,55,59,116,119,120}$ IL-23 can stimulate MAIT cell accumulation in inflamed sites and promote Th17-like properties. $^{31,47}$ MAIT cells also require IL-2 for maximum IFN- $\gamma$ and GzB production as they increase CD25 expression once activated. ${ }^{8,43,58} \mathrm{CD} 14^{+}$monocytes were shown to be a major MAIT cell activating population by being an important source of IL-12 and IL-18 along with activated resident macrophages. ${ }^{18,31,41,44,58,121,122}$ Evidence also suggests potential monocyte stimulation of MAIT cells through IL-1 $\beta .^{31}$ Moreover, costimulatory molecules such as ICOS/ICOS-L, expressed by other immune cells could participate in MAIT cell activation. ${ }^{47}$ Therefore, as discussed earlier, optimal MAIT cell activation is dependent on other immune cells to acquire all their proinflammatory effector capabilities and to proliferate. ${ }^{2,44,47,59,116}$

MAIT cell activation by mucosal cells

Not only professional APCs, but also non-immune cells can activate MAIT cells in a TCR-dependent or independent manner (Fig. 2). Indeed, following infection with M. tuberculosis, S. flexneri, or S. Typhimurium, lung epithelial cells gained MR1 surface expression and were able to directly activate MAIT cells in a TCR-dependent manner. ${ }^{6,47,57,123}$ Of note, one study detailed an MR1 single nucleotide polymorphism in an intronic region that might affect MR1 expression on pulmonary keratinocytes and was associated with higher susceptibility to disseminated tuberculosis. ${ }^{124}$ Gain of MR1 surface expression resulting in MAIT cell activation is also probably at play in the gut mucosa during $H$. pylori infection. ${ }^{10}$ Interestingly, non-invasive S. Typhimurium failed to activate MAIT cells suggesting that cytoplasm invasion of the infected cell is essential for this bacterium to trigger a MAIT cell response. ${ }^{47,57}$ Cancer cells exposing riboflavin-derived ligands on MR1 were also able to activate MAIT cells in a TCR-dependent manner. ${ }^{60,91}$ Cytokine production from epithelial cells, particularly IL-15, may also activate or support MAIT cell activation. ${ }^{125}$

MAIT cells: "bandmaster" of the mucosal immune responses Activated MAIT cells are experts at supporting local immune responses (Fig. 3). They produce IL-17, IL-22, IFN- $\gamma$, TNF- $a$, and iNOS which directly stimulate innate immunity by improving the degradation of phagocyted bacteria by macrophages as well as improving their recruitment in inflamed tissues. $6,12,13,20,24,41,49,58,62$ There is a mutual reinforcement between MAIT cells that stimulate macrophages by producing IFN- $\gamma$, iNOS, TNF- $\alpha$, and macrophages 
supporting MAIT cells through the production of IL-12. MAIT cells can also block intracellular bacteria replication in bone-marrowderived macrophages requiring TCR-MR1 interaction and IL-12. ${ }^{20}$ They also produce IL-2 that stimulates immune cell proliferation as well as Th1 responses, in association with IFN- $\gamma^{21,24,58}$

MAIT cells are therefore critical suppliers of Th1 and Th17 cytokines in inflamed mucosal tissues before the arrival of conventional $\mathrm{CD}^{+}{ }^{+} \mathrm{T}$ cells. MAIT cells are also one major producer of GM-CSF in mucosae during infections and thus support DC maturation. ${ }^{49,64,126} \mathrm{GM}-\mathrm{CSF}$ converts Ly6C ${ }^{\text {high }} \mathrm{CD} 1 \mathrm{~b}^{+}$inflamma-

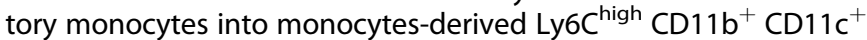
$\mathrm{MHCl}^{+} \mathrm{DC}$ and could therefore improve activation of conventional $\mathrm{CD} 4^{+} \mathrm{T}$ cells and their migration toward the infected lung. ${ }^{64}$ Similarly, during IAV infection, mice lacking MAIT cells displayed reduced numbers of alveolar macrophages and adaptive conventional T cells. ${ }^{43} \mathrm{~A}$ delayed adaptive response, both in recruitment and activation was also observed in MAIT-deficient mice infected with $F$. tularensis. ${ }^{20}$ While the relative frequencies of MAIT cells are reduced as conventional $T$ cells infiltrate the inflamed tissue, they maintain a mixed Th1/Th17 secretion profile and remain in the tissue once infection is resolved ${ }^{8,20,43,49}$ (Tables 1 and 2).

Despite major pro-inflammatory features, more evidence supports now also a potential immunosuppressive function of MAIT cells. Production of IL-10 and TGF- $\beta$ by MAIT cells has been demonstrated in vivo and in vitro. ${ }^{29,70,127}$ However, direct in vivo production notably during the tissue repair process by MAIT cells remains to be demonstrated. ${ }^{14,15}$

MAIT cells: an interesting target to improve mucosal immunity? MAIT cells by their localization and their effector functions can rapidly respond against pathogens and act as sentinels that help recruit adaptive responses. ${ }^{20,43,49,64,128}$ MAIT cells expand, remain at elevated frequencies long after infection and upregulate IL-15 expression that could improve memory $\mathrm{T}$ cell survivability. ${ }^{21,43,47,49,129}$ Activation of MAIT cells correlated with vaccine-induced T-cell responses against adenovirus vaccine platforms and $\mathrm{Mr}^{-/-}$mice displayed reduced responses to vaccine antigens, underlying their implication in effective responses to vaccines. ${ }^{122}$ Therefore, MAIT cells may favor the maintenance of both tissue-resident and central memory $T$ cells in tissues and secondary lymphoid organs. Manipulating MAIT cells by mucosal vaccination challenge might thus be an interesting approach to improve mucosal immunity barrier and reduce risks of pathology.

Several strategies in mice promisingly induced MAIT cell expansion in tissues, especially in the lungs. Oral challenge with S. Typhimurium increased MAIT cell frequency and activation in lungs, associated with phenotypic switches toward an increase in T-bet and IFN- $\gamma$ expression. ${ }^{8,74}$ Multiple groups obtained similar results with synthetic 5-OP-RU associated with TLR2/6 or TLR9 agonist administration, up to an impressive 100-fold MAIT cell expansion. $8,29,49,70,71,74$ Effect was general as intranasal exposure increased MAIT cell frequency in lungs, but also in gut and spleen, although at a lower level. $^{74}$

However, functional results for artificial MAIT cell expansion are mixed in both acute and chronic infections. Indeed, intranasal inoculation of 5-OP-RU and IL-23 resulted in significantly reduced bacterial load after acute infection with $L$. longbeachae, demonstrating positive outcomes of MAIT cell pre-activation with synthetic ligands. ${ }^{47,49}$ In contrast, during $M$. tuberculosis acute infection, 5-OP-RU and CPG vaccination did not modify bacterial loads and disease outcome in the lungs between untreated and MAIT cell-vaccinated WT mice. ${ }^{29,70,71}$ Worse, Sakai and colleagues demonstrated that MAIT cell expansion delayed the conventional $\mathrm{CD}^{+} \mathrm{T}$ cell response. ${ }^{29}$ Thus they showed that TGF- $\beta$ production by MAIT cells reduced in the lung-draining lymph node the number of $\mathrm{Ly}_{6 \mathrm{C}} \mathrm{CCR}^{+}$myeloid cells, which are critical for $\mathrm{CD}^{+}$ $\mathrm{T}$ cell activation. ${ }^{29}$ On the contrary, during chronic tuberculosis with an established inflammatory environment, 5-OP-RU and CpG vaccination drove an increase in Th17 MAIT cell (MAIT17) and Th1 MAIT cell (MAIT1) levels. PD-1 and Ki-67 expression in MAIT cells were increased and taken together, resulted in an IL17A-dependent reduction of pulmonary $M$. tuberculosis bacterial load. $^{29}$

Yet, MAIT cell vaccination strategies might be deleterious in chronic infection where the adaptive response is pathogenic, either directly or via a bystander effect. Indeed, MAIT cells are suspected to be directly involved in several auto-immune diseases. ${ }^{130}$ Priming MAIT cells through oral challenge resulted in increased gastritis in mice with chronic $H$. pylori infection because of increased recruitment of pathogenic inflammatory T cells through MAIT cell activation and expansion. ${ }^{74}$

Overall these contrasting results highlight the complex calibration of MAIT cell activation. Artificially expanding these cells in a sub-inflammatory setting may result, as hypothesized earlier, in promotion of tolerogenic and tissue-repairing functions, while the same strategy applied in chronic, hyperinflammatory conditions may favor aggressive pro-inflammatory MAIT cell functions. ${ }^{29}$ Finally, it is important to stress that almost all the results up to today were obtained in specific pathogen-free mice that have been exposed to very few pathogens during their lives. This is not the case in humans, and strategies based on MAIT cell expansion through vaccination challenge may not be as efficient as for mice that live in clean conditions. More studies are therefore required to assess both the efficiency and the safety of MAIT cell-based vaccination, especially in humans.

\section{CONCLUSION AND PERSPECTIVES}

Although the roles of mucosal MAIT cells are being increasingly characterized, several points should be further explored. First, much remains to fully define their differences in mucosal tissues compared to blood. It will notably be important to define whether MAIT cells display distinct functional profiles depending on the tissue. Second, further studies are required to fully understand the balance between their tissue repair potential and their pro-inflammatory/cytotoxic features, to unveil the mechanisms that control this balance and notably the role of the microbiota. Third, recent work suggests that mucosal MAIT cells may be attractive targets to stimulate through vaccinal approaches. Better understanding of the mechanisms at play would be important before moving to human testing. Nonetheless, much of the data presented here demonstrate that MAIT cells are major actors of the epithelial barrier protection and should be further studied to understand the pathophysiology of mucosal and skin diseases.

\section{ACKNOWLEDGEMENTS}

We are grateful to Matthieu Rouland and Lucie Beaudoin for critical reading of the manuscript. This work was supported by grants from INSERM, CNRS, Laboratoire d'Excellence consortium Inflamex (grant number ANR-11-IDEX-0005-02), Agence Nationale de la Recherche (ANR-17-CE14-0002-01 Diab1MAIT, ANR-19-CE14-0041-01 HEPADIMAIT), RHU QUID-NASH ANR-17-RHUS-009, Fondation pour la Recherche Médicale (grant number EQU201903007779), EFSD/JDRF/Lilly to A.L., EFSD/Lilly grant to A.L. and A.T., an Aide aux Jeunes Diabétiques fellowship to I.N. and a Ministry of Research PhD grant to L.B. This study has been also granted from the FFRD, sponsored by Fédération Française des Diabétiques (FFD), Abbott, AstraZeneca, Eli Lilly, Merck Sharp \& Dohme (MSD) and Novo Nordisk.

\section{AUTHOR CONTRIBUTIONS}

I.N., L.B., A.T. and A.L. designed figures, tables, and wrote the present review. 


\section{ADDITIONAL INFORMATION}

Competing interests: The authors declare no competing interests.

Publisher's note Springer Nature remains neutral with regard to jurisdictional claims in published maps and institutional affiliations.

\section{REFERENCES}

1. Porcelli, S., Yockey, C. E., Brenner, M. B. \& Balk, S. P. Analysis of T cell antigen receptor (TCR) expression by human peripheral blood CD4-8- alpha/beta T cells demonstrates preferential use of several $\mathrm{V}$ beta genes and an invariant TCR alpha chain. J. Exp. Med. 178, 1-16 (1993).

2. Dusseaux, M. et al. Human MAIT cells are xenobiotic-resistant, tissue-targeted, CD161 hi IL-17-secreting T cells. Blood 117, 1250-1259 (2011).

3. Reantragoon, R. et al. Antigen-loaded MR1 tetramers define T cell receptor heterogeneity in mucosal-associated invariant T cells. J. Exp. Med. 210, 2305-2320 (2013).

4. Hegde, P. et al. Mucosal-associated invariant T cells are a profibrogenic immune cell population in the liver. Nat. Commun. 9, 2146 (2018).

5. Sobkowiak, M. J. et al. Tissue-resident MAIT cell populations in human oral mucosa exhibit an activated profile and produce IL-17. Eur. J. Immunol. 49, 133-143 (2019).

6. Gold, M. C. et al. Human mucosal associated invariant T cells detect bacterially infected cells. PLoS Biol. 8, e1000407 (2010).

7. Rahimpour, A. et al. Identification of phenotypically and functionally heterogeneous mouse mucosal-associated invariant T cells using MR1 tetramers. J. Exp. Med. 212, 1095-1108 (2015).

8. Chen, Z. et al. Mucosal-associated invariant T-cell activation and accumulation after in vivo infection depends on microbial riboflavin synthesis and costimulatory signals. Mucosal Immunol. 10, 58-68 (2017).

9. Treiner, E. et al. Selection of evolutionarily conserved mucosal-associated invariant T cells by MR1. Nature 422, 164-169 (2003).

10. Booth, J. S. et al. Mucosal-associated invariant $T$ cells in the human gastric mucosa and blood: role in Helicobacter pylori infection. Front. Immunol. 6, 466 (2015).

11. Magalhaes, I. et al. Mucosal-associated invariant $\mathrm{T}$ cell alterations in obese and type 2 diabetic patients. J. Clin. Invest. 125, 1752-1762 (2015).

12. Rouxel, O. et al. Cytotoxic and regulatory roles of mucosal-associated invariant T cells in type 1 diabetes. Nat. Immunol. 18, 1321-1331 (2017).

13. Gibbs, A. et al. MAIT cells reside in the female genital mucosa and are biased towards IL-17 and IL-22 production in response to bacterial stimulation. Mucosal Immunol. 10, 35-45 (2017).

14. Constantinides, M. G. et al. MAIT cells are imprinted by the microbiota in early life and promote tissue repair. Science 366, eaax6624 (2019).

15. Leng, T. et al. TCR and inflammatory signals tune human MAIT cells to exert specific tissue repair and effector functions. Cell Rep. 28, 3077-3091.e5 (2019).

16. Martin, E. et al. Stepwise development of MAIT cells in mouse and human. PLoS Biol. 7, e54 (2009).

17. Tilloy, F. et al. An invariant $\mathrm{T}$ cell receptor alpha chain defines a novel TAPindependent major histocompatibility complex class lb-restricted alpha/beta $T$ cell subpopulation in mammals. J. Exp. Med. 189, 1907-1921 (1999).

18. Flament, $\mathrm{H}$. et al. Outcome of SARS-CoV-2 infection is linked to MAIT cell activation and cytotoxicity. Nat. Immunol. 22, 322-335, https://doi.org/10.1038/ s41590-021-00870-z (2021).

19. Jouan, Y. et al. Phenotypical and functional alteration of unconventional T cells in severe COVID-19 patients. J. Exp. Med. 217, e20200872 (2020).

20. Meierovics, A., Yankelevich, W.-J. C. \& Cowley, S. C. MAIT cells are critical for optimal mucosal immune responses during in vivo pulmonary bacterial infection. Proc. Natl Acad. Sci. USA. 110, E3119-E3128 (2013).

21. Hinks, T. S. C. et al. Activation and in vivo evolution of the MAIT cell transcriptome in mice and humans reveals tissue repair functionality. Cell Rep. 28, 3249-3262.e5 (2019).

22. Salou, M. et al. A common transcriptomic program acquired in the thymus defines tissue residency of MAIT and NKT subsets. J. Exp. Med. 216, 133-151 (2019).

23. Gherardin, N. A. et al. Human blood MAIT cell subsets defined using MR1 tetramers. Immunol. Cell Biol. 96, 507-525 (2018).

24. Sakala, I. G. et al. Functional heterogeneity and antimycobacterial effects of mouse mucosal-associated invariant T cells specific for riboflavin metabolites. J. Immunol. 195, 587-601 (2015).

25. Godfrey, D. I., Koay, H.-F., McCluskey, J. \& Gherardin, N. A. The biology and functional importance of MAIT cells. Nat. Immunol. 20, 1110-1128 (2019).

26. Toubal, A., Nel, I., Lotersztajn, S. \& Lehuen, A. Mucosal-associated invariant T cells and disease. Nat. Rev. Immunol. 19, 643-657 (2019).
27. Provine, N. M. \& Klenerman, P. MAIT cells in health and disease. Annu. Rev. Immunol. https://doi.org/10.1146/annurev-immunol-080719-015428 (2020).

28. Salerno-Goncalves, R. et al. Challenge of humans with wild-type salmonella enterica serovar typhi elicits changes in the activation and homing characteristics of mucosal-associated invariant T cells. Front. Immunol. 8, 398 (2017).

29. Sakai, S. et al. MAIT cell-directed therapy of Mycobacterium tuberculosis infection. Mucosal Immunol. https://doi.org/10.1038/s41385-020-0332-4 (2020).

30. Greene, J. M. et al. MR1-restricted mucosal-associated invariant T (MAIT) cells respond to mycobacterial vaccination and infection in nonhuman primates. Mucosal Immunol. 10, 802-813 (2017).

31. Lu, B. et al. IL-17 production by tissue-resident MAIT cells is locally induced in children with pneumonia. Mucosal Immunol. 13, 824-835 (2020).

32. Li, J. et al. The frequency of mucosal-associated invariant T cells is selectively increased in dermatitis herpetiformis. Australas. J. Dermatol. 58, 200-204 (2017).

33. Voillet, V. et al. Human MAIT cells exit peripheral tissues and recirculate via lymph in steady state conditions. JCl Insight 3, e98487 (2018).

34. Kjer-Nielsen, L. et al. MR1 presents microbial vitamin B metabolites to MAIT cells. Nature 491, 717-723 (2012).

35. Corbett, A. J. et al. T-cell activation by transitory neo-antigens derived from distinct microbial pathways. Nature 509, 361-365 (2014).

36. Patel, O. et al. Recognition of vitamin B metabolites by mucosal-associated invariant T cells. Nat. Commun. 4, 2142 (2013).

37. Eckle, S. B. G. et al. A molecular basis underpinning the T cell receptor heterogeneity of mucosal-associated invariant T cells. J. Exp. Med. 211, 1585-1600 (2014).

38. Ussher, J. E. et al. CD161++ CD8+ T cells, including the MAIT cell subset, are specifically activated by IL-12+IL-18 in a TCR-independent manner. Eur. J. Immunol. 44, 195-203 (2014).

39. Leeansyah, E. et al. Arming of MAIT cell cytolytic antimicrobial activity is induced by IL-7 and defective in HIV-1 infection. PLoS Pathog. 11, e1005072 (2015).

40. Sattler, A., Dang-Heine, C., Reinke, P. \& Babel, N. IL-15 dependent induction of IL18 secretion as a feedback mechanism controlling human MAIT-cell effector functions. Eur. J. Immunol. 45, 2286-2298 (2015).

41. Loh, $\mathrm{L}$. et al. Human mucosal-associated invariant T cells contribute to antiviral influenza immunity via IL-18-dependent activation. Proc. Natl Acad. Sci. USA. 113, 10133-10138 (2016)

42. Wilgenburg, Bvan et al. MAIT cells are activated during human viral infections. Nat. Commun. 7, 11653 (2016).

43. Wilgenburg, Bvan et al. MAIT cells contribute to protection against lethal influenza infection in vivo. Nat. Commun. 9, 4706 (2018).

44. Kurioka, A. et al. Diverse streptococcus pneumoniae strains drive a mucosalassociated invariant T-cell response through major histocompatibility complex class I-related molecule-dependent and cytokine-driven pathways. J. Infect. Dis. 217, 988-999 (2018).

45. Le Bourhis, L. et al. Antimicrobial activity of mucosal-associated invariant T cells. Nat. Immunol. 11, 701-708 (2010).

46. Sundström, P. et al. Human mucosa-associated invariant $T$ cells accumulate in colon adenocarcinomas but produce reduced amounts of IFN- $\gamma$. J. Immunol. 195, 3472-3481 (2015)

47. Wang, $\mathrm{H}$. et al. IL-23 costimulates antigen-specific MAIT cell activation and enables vaccination against bacterial infection. Sci. Immunol. 4, eaaw0402 (2019).

48. Schmaler, M. et al. Modulation of bacterial metabolism by the microenvironment controls MAIT cell stimulation. Mucosal Immunol. 11, 1060-1070 (2018).

49. Wang, $H$. et al. MAIT cells protect against pulmonary Legionella longbeachae infection. Nat. Commun. 9, 3350 (2018).

50. $\mathrm{Yu}, \mathrm{H}$. et al. CXCL16 stimulates antigen-induced MAIT cell accumulation but trafficking during lung infection Is CXCR6-independent. Front. Immunol. 11, 1773 (2020).

51. Serriari, N.-E. et al. Innate mucosal-associated invariant T (MAIT) cells are activated in inflammatory bowel diseases. Clin. Exp. Immunol. 176, 266-274 (2014).

52. Mestan, J. et al. Antiviral effects of recombinant tumour necrosis factor in vitro. Nature 323, 816-819 (1986).

53. Samuel, C. E. Antiviral actions of interferons. Clin. Microbiol. Rev. 14, 778-809 (2001).

54. Braumüller, $H$. et al. T-helper-1-cell cytokines drive cancer into senescence. Nature 494, 361-365 (2013)

55. Kurioka, A. et al. MAIT cells are licensed through granzyme exchange to kill bacterially sensitized targets. Mucosal Immunol. 8, 429-440 (2015).

56. Lamichhane, R. et al. TCR- or cytokine-activated CD8+ mucosal-associated invariant $\mathrm{T}$ cells are rapid polyfunctional effectors that can coordinate immune responses. Cell Rep. 28, 3061-3076.e5 (2019).

57. Le Bourhis, L. et al. MAIT cells detect and efficiently lyse bacterially-infected epithelial cells. PLoS Pathog. 9, e1003681 (2013). 
58. Jiang, J. et al. Enhanced immune response of MAIT cells in tuberculous pleural effusions depends on cytokine signaling. Sci. Rep. 6, 32320 (2016).

59. Wallington, J. C., Williams, A. P., Staples, K. J. \& Wilkinson, T. M. A. IL-12 and IL-7 synergize to control mucosal-associated invariant T-cell cytotoxic responses to bacterial infection. J. Allergy Clin. Immunol. 141, 2182-2195.e6 (2018).

60. Won, E. J. et al. Clinical relevance of circulating mucosal-associated invariant $T$ cell levels and their anti-cancer activity in patients with mucosal-associated cancer. Oncotarget 7, 76274-76290 (2016).

61. Georgel, P., Radosavljevic, M., Macquin, C. \& Bahram, S. The non-conventional MHC class I MR1 molecule controls infection by Klebsiella pneumoniae in mice. Mol. Immunol. 48, 769-775 (2011).

62. Chua, W.-J. et al. Polyclonal mucosa-associated invariant $\mathrm{T}$ cells have unique innate functions in bacterial infection. Infect. Immun. 80, 3256-3267 (2012).

63. Cui, Y. et al. Mucosal-associated invariant $T$ cell-rich congenic mouse strain allows functional evaluation. J. Clin. Invest. 125, 4171-4185 (2015).

64. Meierovics, A. I. \& Cowley, S. C. MAIT cells promote inflammatory monocyte differentiation into dendritic cells during pulmonary intracellular infection. J. Exp. Med. 213, 2793-2809 (2016).

65. Jahreis, S. et al. Human MAIT cells are rapidly activated by Aspergillus spp. in an APC-dependent manner. Eur. J. Immunol. 48, 1698-1706 (2018).

66. Jesteadt, E. et al. Interleukin-18 is critical for mucosa-associated invariant T cell gamma interferon responses to francisella species in vitro but not in vivo. Infect. Immun. 86, e00117-18 (2018).

67. Kwon, Y.-S. et al. Mucosal-associated invariant T cells are numerically and functionally deficient in patients with mycobacterial infection and reflect disease activity. Tuberculosis 95, 267-274 (2015).

68. Howson, L. J. et al. MAIT cell clonal expansion and TCR repertoire shaping in human volunteers challenged with Salmonella Paratyphi A. Nat. Commun. 9, 253 (2018).

69. Grimaldi, D. et al. Specific MAIT cell behaviour among innate-like T lymphocytes in critically ill patients with severe infections. Intensive Care Med. 40, 192-201 (2014).

70. $\mathrm{Yu}, \mathrm{H}$. et al. Artificially induced MAIT cells inhibit M. bovis BCG but not M. tuberculosis during in vivo pulmonary infection. Sci. Rep. 10, 13579 (2020)

71. Vorkas, C. K. et al. Efficient 5-OP-RU-induced enrichment of Mucosal-associated invariant $T$ cells in the murine lung does not enhance control of aerosol Mycobacterium tuberculosis infection. Infect. Immun. https://doi.org/10.1128/ IAI.00524-20 (2020).

72. Howson, L. J. et al. Absence of mucosal-associated invariant T cells in a person with a homozygous point mutation in MR1. Sci. Immunol. 5, eabc9492 (2020).

73. Smith, A. D. et al. Microbiota of MR1 deficient mice confer resistance against Clostridium difficile infection. PLOS ONE 14, e0223025 (2019).

74. D'Souza, C. et al. Mucosal-associated invariant $T$ cells augment immunopathology and gastritis in chronic Helicobacter pylori infection. J. Immunol. 200, 1901-1916 (2018)

75. Ussher, J. E., Willberg, C. B. \& Klenerman, P. MAIT cells and viruses. Immunol. Cell Biol. 96, 630-641 (2018).

76. Cosgrove, C. et al. Early and nonreversible decrease of CD161++ /MAIT cells in HIV infection. Blood 121, 951-961 (2013).

77. Leeansyah, E. et al. Activation, exhaustion, and persistent decline of the antimicrobial MR1-restricted MAIT-cell population in chronic HIV-1 infection. Blood 121, 1124-1135 (2013).

78. Greathead, L. et al. CD8+/CD161++ mucosal-associated invariant T-cell levels in the colon are restored on long-term antiretroviral therapy and correlate with CD8+ T-cell immune activation. AIDS 28, 1690-1692 (2014).

79. Magnoumba, M. et al. Unbiased profiling reveals compartmentalization of unconventional T-cells within the intestinal mucosa irrespective of HIV infection. Front Immunol. 11, 579743 (2020).

80. Parrot, T. et al. MAIT cell activation and dynamics associated with COVID-19 disease severity. Sci. Immunol. 5, eabe1670 (2020).

81. Rudak, P. T., Yao, T., Richardson, C. D. \& Haeryfar, S. M. M. Measles virus infects and programs MAIT cells for apoptosis. J. Infect. Dis. https://doi.org/10.1093/ infdis/jiaa407 (2020).

82. McSharry, B. P. et al. Virus-mediated suppression of the antigen presentation molecule MR1. Cell Rep. 30, 2948-2962.e4 (2020).

83. Sausset, R., Petit, M. A., Gaboriau-Routhiau, V. \& De Paepe, M. New insights into intestinal phages. Mucosal Immunol. 13, 205-215 (2020)

84. Van Belleghem, J. D., Dąbrowska, K., Vaneechoutte, M., Barr, J. J. \& Bollyky, P. L. Interactions between bacteriophage, bacteria, and the mammalian immune system. Viruses 11, 10 (2018).

85. Melo, A. M. et al. Mucosal-associated invariant T cells display diminished effector capacity in oesophageal adenocarcinoma. Front. Immunol. 10, 1580 (2019).

86. Ling, L. et al. Circulating and tumor-infiltrating mucosal associated invariant $T$ (MAIT) cells in colorectal cancer patients. Sci. Rep. 6, 20358 (2016).
87. Zabijak, L. et al. Increased tumor infiltration by mucosal-associated invariant $\mathrm{T}$ cells correlates with poor survival in colorectal cancer patients. Cancer Immunol. Immunother. 64, 1601-1608 (2015).

88. Sundström, P. et al. Tumor-infiltrating mucosal-associated invariant T (MAIT) cells retain expression of cytotoxic effector molecules. Oncotarget 10, 2810-2823 (2019)

89. Berzins, S. P., Wallace, M. E., Kannourakis, G. \& Kelly, J. A role for MAIT cells in colorectal cancer. Front. Immunol. 11, 949 (2020).

90. Shaler, C. R. et al. Mucosa-associated invariant T cells infiltrate hepatic metastases in patients with colorectal carcinoma but are rendered dysfunctional within and adjacent to tumor microenvironment. Cancer Immunol. Immunother. 66, 1563-1575 (2017)

91. Yan, J. et al. MAIT cells promote tumor initiation, growth, and metastases via tumor MR1. Cancer Discov. 10, 124-141 (2020).

92. Forbes, S. J. \& Rosenthal, N. Preparing the ground for tissue regeneration: from mechanism to therapy. Nat. Med. 20, 857-869 (2014).

93. Dudakov, J. A., Hanash, A. M. \& Brink, M. R. Mvanden Interleukin-22: immunobiology and pathology. Annu. Rev. Immunol. 33, 747-785 (2015).

94. Sugimoto, K. et al. IL-22 ameliorates intestinal inflammation in a mouse model of ulcerative colitis. J. Clin. Invest. 118, 534-544 (2008).

95. Lee, J. S. et al. Interleukin-23-independent IL-17 production regulates intestinal epithelial permeability. Immunity 43, 727-738 (2015).

96. Varelias, A. et al. Recipient mucosal-associated invariant T cells control GVHD within the colon. J. Clin. Invest. 128, 1919-1936 (2018).

97. Linehan, J. L. et al. Non-classical immunity controls microbiota impact on skin immunity and tissue repair. Cell 172, 784-796.e18 (2018).

98. Rouland, M. et al. Gut mucosa alterations and loss of segmented filamentous bacteria in type 1 diabetes are associated with inflammation rather than hyperglycemia. Gut 1-13, https://doi.org/10.1136/gutjnl-2020-323664 (2021).

99. Maxwell, J. R. et al. Differential roles for interleukin-23 and interleukin-17 in intestinal immunoregulation. Immunity 43, 739-750 (2015).

100. Eyerich, K., Dimartino, V. \& Cavani, A. IL-17 and IL-22 in immunity: driving protection and pathology. Eur. J. Immunol. 47, 607-614 (2017).

101. Toubal, A. et al. Mucosal-associated invariant T cells promote inflammation and intestinal dysbiosis leading to metabolic dysfunction during obesity. Nat. Commun. 11, 3755 (2020)

102. Bertrand, L. \& Lehuen, A. MAIT cells in metabolic diseases. Mol. Metab. 27S, S114-S121 (2019)

103. Haga, K. et al. MAIT cells are activated and accumulated in the inflamed mucosa of ulcerative colitis. J. Gastroenterol. Hepatol. 31, 965-972 (2016).

104. Hiejima, E. et al. Reduced numbers and proapoptotic features of mucosalassociated invariant $T$ cells as a characteristic finding in patients with inflammatory bowel disease. Inflamm. Bowel Dis. 21, 1529-1540 (2015).

105. Molet, S. et al. IL-17 is increased in asthmatic airways and induces human bronchial fibroblasts to produce cytokines. J. Allergy Clin. Immunol. 108, 430-438 (2001).

106. Korn, T., Bettelli, E., Oukka, M. \& Kuchroo, V. K. IL-17 and Th17 Cells. Annu. Rev. Immunol. 27, 485-517 (2009).

107. Kulig, P. et al. IL17A-mediated endothelial breach promotes metastasis formation. Cancer Immunol. Res. 4, 26-32 (2016).

108. DeAngelis, L. M., Cirillo, N. \& McCullough, M. J. The immunopathogenesis of oral lichen planus-ls there a role for mucosal associated invariant T cells? J. Oral. Pathol. Med. 48, 552-559 (2019).

109. Salou, M. \& Lantz, O. A TCR-dependent tissue repair potential of MAIT cells. Trends Immunol. 40, 975-977 (2019).

110. Böttcher, K. et al. MAIT cells are chronically activated in patients with autoimmune liver disease and promote profibrogenic hepatic stellate cell activation. Hepatology 68, 172-186 (2018).

111. Berg, R. D. Bacterial translocation from the gastrointestinal tract. Adv. Exp. Med. Biol. 473, 11-30 (1999).

112. Tastan, $C$. et al. Tuning of human MAIT cell activation by commensal bacteria species and MR1-dependent T-cell presentation. Mucosal Immunol. 11, 1591-1605 (2018).

113. Krause, J. L. et al. The activation of mucosal-associated invariant T (MAIT) cells is affected by microbial diversity and riboflavin utilization in vitro. Front. Microbiol. 11, 755 (2020).

114. Slichter, C. K. et al. Distinct activation thresholds of human conventional and innate-like memory T cells. JCl Insight 1, e86292 (2016).

115. Jenkins, M. K., Pardoll, D. M., Mizuguchi, J., Quill, H. \& Schwartz, R. H. T-cell unresponsiveness in vivo and in vitro: fine specificity of induction and molecular characterization of the unresponsive state. Immunol. Rev. 95, 113-135 (1987).

116. Pavlovic, M., Gross, C., Chili, C., Secher, T. \& Treiner, E. MAIT cells display a specific response to type 1 IFN underlying the adjuvant effect of TLR7/8 ligands. Front. Immunol. 11, 2097 (2020). 
117. Hartmann, N. et al. Riboflavin metabolism variation among clinical isolates of streptococcus pneumoniae results in differential activation of mucosalassociated invariant T cells. Am. J. Respir. Cell Mol. Biol. 58, 767-776 (2018).

118. Ussher, J. E. et al. TLR signaling in human antigen-presenting cells regulates MR1-dependent activation of MAIT cells. Eur. J. Immunol. 46, 1600-1614 (2016).

119. Lamichhane, R. et al. Type I interferons are important co-stimulatory signals during T cell receptor mediated human MAIT cell activation. Eur. J. Immunol. 50, 178-191 (2020).

120. Gracey, E. et al. IL-7 primes IL-17 in mucosal-associated invariant T (MAIT) cells, which contribute to the Th17-axis in ankylosing spondylitis. Ann. Rheum. Dis. 75, 2124-2132 (2016).

121. Jo, J. et al. Toll-like receptor 8 agonist and bacteria trigger potent activation of innate immune cells in human liver. PLoS Pathog. 10, e1004210 (2014)

122. Provine, N. M. et al. MAIT cell activation augments adenovirus vector vaccine immunogenicity. Science 371, 521-526 (2021)

123. Harriff, M. J. et al. Endosomal MR1 trafficking plays a key role in presentation of mycobacterium tuberculosis ligands to MAIT cells. PLoS Pathog. 12, e1005524 (2016).

124. Seshadri, C. et al. A polymorphism in human MR1 is associated with mRNA expression and susceptibility to tuberculosis. Genes Immun. 18, 8-14 (2017).

125. Reinecker, H., MacDermott, R., Mirau, S., Dignass, A. \& Podolsky, D. Intestinal epithelial cells both express and respond to interleukin 15. Gastroenterology 111, 1706-1713 (1996)
126. Salio, M. et al. Activation of human mucosal-associated invariant $T$ cells induces CD40L-dependent maturation of monocyte-derived and primary dendritic cells. J. Immunol. 199, 2631-2638 (2017).

127. Croxford, J. L., Miyake, S., Huang, Y.-Y., Shimamura, M. \& Yamamura, T. Invariant V(alpha)19i T cells regulate autoimmune inflammation. Nat. Immunol. 7, 987-994 (2006).

128. Berkson, J. D. \& Prlic, M. The MAIT conundrum-how human MAIT cells distinguish bacterial colonization from infection in mucosal barrier tissues. Immunol. Lett. 192, 7-11 (2017).

129. Berard, M., Brandt, K., Bulfone-Paus, S. \& Tough, D. F. IL-15 promotes the survival of naive and memory phenotype CD8+ T cells. J. Immunol. 170, 5018-5026 (2003).

130. Rouxel, O. \& Lehuen, A. Mucosal-associated invariant T cells in autoimmune and immune-mediated diseases. Immunol. Cell Biol. 96, 618-629 (2018).

131. Tominaga, K. et al. Possible involvement of mucosal-associated invariant $T$ cells in the progression of inflammatory bowel diseases. Biomed. Res. 38, 111-121 (2017).

132. Juno, J. A. et al. MAIT cells upregulate $\alpha 4 \beta 7$ in response to acute simian immunodeficiency virus/simian HIV infection but are resistant to peripheral depletion in pigtail macaques. J. Immunol. 202, 2105-2120 (2019).

133. Kauffman, K. D. et al. Limited pulmonary mucosal-associated invariant $\mathrm{T}$ cell accumulation and activation during Mycobacterium tuberculosis infection in rhesus macaques. Infect. Immun. 86, e00431-18 (2018). 